Niklas Nilsson*, Katerina Nezvalova-Henriksen and Ingunn Tho

\title{
Emulsion Stability of Different Intravenous Propofol Formulations in Simulated Co-Administration with Remifentanil Hydrochloride
}

https://doi.org/10.1515/pthp-2019-0014

Received June 03, 2019; revised July 05, 2019; accepted July 05, 2019

\section{Abstract}

Background: Propofol and remifentanil often need to be co-administered via the same intravenous catheter line, which predisposes to potential compatibility issues. Our aim was to determine and compare the emulsion stability of three propofol formulations, two with medium chain triglycerides and one with long chain triglycerides, when administered together with remifentanil hydrochloride.

Methods: Remifentanil hydrochloride (Ultiva ${ }^{\circledR}$ ) $50 \mu \mathrm{g} / \mathrm{mL}$ was mixed with two concentrations (10 and $20 \mathrm{mg} / \mathrm{mL}$ ) of each propofol formulation in mixing ratios $10+1,20+1$, $1+1$ and $1+20$. Emulsion stability was assessed immediately after mixing and 4 hours later by measurements of $\mathrm{pH}$, mean droplet diameter, polydispersity index, and calculating percentage of fat residing in globules $>5 \mu \mathrm{m}$ (PFAT5).

Results: High PFAT5 values were observed in certain mixing ratios. The correlation between elevated PFAT5 and high propofol concentration $(20 \mathrm{mg} / \mathrm{mL})$, when remifentanil was in abundance and for long contact time indicated that these factors influenced the stability of the propofol emulsions.

Conclusions: Stability differences between the propofol formulations were identified under extreme test conditions. Co-administration of remifentanil and propofol in

\footnotetext{
*Corresponding author: Niklas Nilsson, Department of Pharmacy, Faculty of Mathematics and Natural Sciences, University of Oslo, P.O.Box 1068 Blindern, Oslo 0371, Norway; Oslo Hospital Pharmacy, Rikshospitalet, Norway, E-mail: Niklas.nilsson@farmasi.uio.no; Niklas.nilsson@sykehusapotekene.no https://orcid.org/0000-0002-2047-8058

Katerina Nezvalova-Henriksen, Oslo Hospital Pharmacy, Rikshospitalet, Hospital Pharmacy Enterprise, Oslo 0372, Norway, E-mail: katerina.nezvalova.henriksen@sykehusapotekene.no Ingunn Tho, Department of Pharmacy, Faculty of Mathematics and Natural Sciences, University of Oslo, P.O.Box 1068 Blindern, Oslo 0371, Norway, E-mail: ingunn.tho@farmasi.uio.no
}

the same i.v. line is safe when propofol is in abundance. Caution is advised when remifentanil is present in equal parts or in abundance when co-administered with propofol $20 \mathrm{mg} / \mathrm{mL}$.

Keywords: compatibility, safe intravenous administration, anesthesia, droplet growth, destabilisation, PFAT5

\section{Introduction}

Propofol is a general anesthetic with a rapid onset of action and a short recovery period. It is often used together with remifentanil, an opioid analgesic, to induce and maintain anesthesia or sedation. Propofol is formulated as an oil-in-water emulsion because of its low aqueous solubility (Table 1).

It is a common clinical practice that remifentanil and propofol are co-administered via the same intravenous (i.v.) catheter line. Co-administration of drugs predisposes to potential compatibility issues. Adverse consequences range from i.v. line obstruction to life-threatening embolism. In the case of parenteral emulsions, such as propofol, an incompatibility reaction could manifest as destabilisation of the emulsion. This involves an increase in the diameter size of the propofol oil droplets that ultimately leads to coalescence and phase separation. An early sign of emulsion destabilisation is an increase in droplet size in the large diameter tail of the size distribution [1]. Droplets that reach a diameter of $5 \mu \mathrm{m}$ or more have an increased tendency to become entrapped in small capillaries, for instance those found in the lungs or the brain, and they may cause embolism [2-5]. Entrapment in the liver, the reticuloendothelial system and several other organs has also been reported [6, 7]. In addition, incompatibilities have been suspected of having a deleterious immunomodulating effect $[8,9]$.

The available propofol formulations differ in lipid and excipient composition (Table 2). Our hypothesis is that different propofol formulations may behave differently upon contact with remifentanil.

In clinical practice, infusion flow rates are constantly adjusted to achieve the desired clinical response. 
Table 1: Molecular structure and relevant physico-chemical properties of propofol and remifentanil.

\begin{tabular}{l}
\hline Property \\
Structure
\end{tabular}

Table 2: Overview of the compositions of the investigated propofol formulations (Source: SmPC).

\begin{tabular}{|c|c|c|c|}
\hline Acronym & P-MCT1 & P-MCT2 & $P-L C T$ \\
\hline Product name Manufacturer & $\begin{array}{l}\text { Propolipid }^{\circledR} \\
\text { Fresenius- } \\
\text { Kabi }\end{array}$ & $\begin{array}{l}\text { Propofol- } \\
\text { Lipuro }^{\circledR} \\
\text { B.Braun }\end{array}$ & $\begin{array}{l}\text { Diprivan }^{\circledR} \\
\text { Aspen }\end{array}$ \\
\hline Soya-bean oil (LCT) & $50 \%$ & $50 \%$ & $100 \%$ \\
\hline $\begin{array}{l}\text { Medium-chain triglycerides } \\
\text { (MCT) }\end{array}$ & $50 \%$ & $50 \%$ & \\
\hline Glycerol & + & + & + \\
\hline Purified egg phosphatide & + & & + \\
\hline Egg lecithin & & + & \\
\hline Disodium edetate & & & + \\
\hline Sodium oleate & & + & \\
\hline Oleic acid & + & & \\
\hline Sodium hydroxide & + & & + \\
\hline Water for injections & + & + & + \\
\hline $\mathrm{pH}$ & $7.5-8.5$ & $6.0-8.5$ & $7.0-8.5$ \\
\hline
\end{tabular}

This creates an ever-changing relationship between the drugs that are co-administered via the same i.v. line. It is therefore important to examine these drugs in mixing ratios that mimic the relationships that might occur in the i.v. line during infusion. To the best of our knowledge, there is a lack of thorough compatibility studies comparing propofol formulations after mixing with remifentanil [10-12]. In the case of propofol, an incompatibility can be revealed by a change in the emulsion droplet size. An increase in the content of large sized oil droplets $(>5 \mu \mathrm{m})$ may become critical (exceed the acceptable safety limit) long before it is possible to visually detect emulsion destabilisation.
The aim of this study was to determine and compare the emulsion stability of the three propofol formulations currently used in Norwegian hospitals (Propolipid ${ }^{\circledR}$ (P-MCT1), Propofol-Lipuro $^{\circledR}$ (P-MCT2) and Diprivan (P-LCT)) when administered together with remifentanil in the same i.v. line.

\section{Material and methods}

\section{Materials}

The three commercially available propofol formulations used in this study were Propolipid (Fresenius-Kabi, Bad Homburg, Germany), Propofol-Lipuro (B.Braun Melsungen AG, Melsungen, Germany), Diprivan (Aspen Pharma, Dublin, Ireland). They were used at concentrations of $10 \mathrm{mg} / \mathrm{mL}$ and $20 \mathrm{mg} / \mathrm{mL}$. Remifentanil hydrochloride, Ultiva (GlaxoSmithKline, Brentford, United Kingdom), $2 \mathrm{mg}$ and $5 \mathrm{mg}$ powder for solution for injection/infusion was used.

Water, unless otherwise specified, was purified by the Milli- $Q^{\circledR}$ integrated water purification system for ultrapure water (Elix ${ }^{\circledR}$, Merck Millipore, Darmstadt, Germany) with $0.22 \mu \mathrm{m}$ filtration and from now on referred to as MilliQwater in this manuscript.

\section{Sample preparation}

All sample preparation was done aseptically and in a vertical laminar flow bench. Remifentanil powder was 
reconstituted in $9 \mathrm{mg} / \mathrm{mL}$ sodium chloride to a final concentration of $50 \mu \mathrm{g} / \mathrm{mL}$. The calculated volume of remifentanil was transferred to $50 \mathrm{~mL}$ sterile polypropylene centrifuge tubes with CentriStar ${ }^{\mathrm{TM}}$ cap (VWR, Radnor, Pennsylvania, USA/Coring Incorporated, New York, New York, USA) via a $0.2 \mu \mathrm{m}$ sterile syringe filter (Pall, VWR, Radnor, Pennsylvania, USA). The propofol glass container was carefully shaken prior to extraction of the calculated volume, which was transferred to the centrifuge tube containing remifentanil using a Soft-ject ${ }^{\circledR}$ syringe (Henke-Sass Wolf GmbH, Tuttlingen, Germany).

Remifentanil was mixed with propofol formulations in the following volume per volume $(\mathrm{v} / \mathrm{v})$ mixing ratios respectively: $10+1,20+1,1+1$ and $1+20$. The mixing ratios were chosen to represent the typical drug flow rates in the i.v. line. Each mixing ratio was investigated in three parallels to ensure reproducibility of the results. Control samples consisted of propofol only.

It should be noted that the background level of particle contamination (noise) must be kept at a minimum. This was achieved by selecting tubes and containers with low particle numbers, by rinsing with $0.2 \mu \mathrm{m}$ filtered MilliQ-water and by always measuring the background noise of the specific batch of MilliQ-water employed for the analyses. The acceptance limit for background noise was set to 50 particles/mL as measured by light obscuration.

\section{Stability analysis of the emulsion}

All samples were analysed immediately after mixing and after 4 hours. Emulsion stability was assessed by:

a. measuring the $\mathrm{pH}$

b. measuring mean droplet diameter (MDD) and polydispersity index (PI) of the droplet size distribution by dynamic light scattering (DLS)

c. measuring the number and size of oil droplets larger than $1 \mu \mathrm{m}$ by light obscuration

d. calculation of the percentage of fat droplets larger than $5 \mu \mathrm{m}$ (PFAT5)

The potential change in the stability of each propofol formulation as a result of mixing with remifentanil was assessed by comparing all the characteristics of the reminfentanil-propofol mixtures, with those of propofol alone (the control).

\section{pH measurements}

The $\mathrm{pH}$ was measured for each sample using a $\mathrm{pH}$ meter (Mettler Toledo, Columbus, Ohio, USA) at 0 hours and after 4 hours. The pH-meter was calibrated daily with $\mathrm{pH} 2.00,4.00$ and 9.00 buffers to cover the full range of $\mathrm{pH}$-values encountered.

\section{Dynamic light scattering}

The intensity weighted (I.W.) MDD and PI was determined by DLS with a Zetasizer nano series (Malvern instruments, Malvern, UK). The United States Pharmacopeia (USP) states that a stable i.v. emulsion should not contain droplets with an I.W. MDD of more than $500 \mathrm{~nm}$ [1]. PI is a measure of how uniform the size of the emulsion droplets is and ranges from 0 to 1 . Values below 0.10 indicate a monodisperse size distribution (uniform droplet size) consistent with a stable emulsion, whereas values above 0.50 are indicative of a very broad size distribution, which is less desirable with respect to emulsion stability. The latter could suggest an increasing droplet size caused by droplet growth [13]. DLS is suitable for measurement of particles in the size range of nm up to maximally $1 \mu \mathrm{m}$.

The droplet concentration of propofol emulsions and mixed samples were too high to produce reliable measurements, and the samples were diluted 58-801 times (sample:water) in prefiltered $0.2 \mu \mathrm{m}$ (Pall, VWR, Radnor, Pennsylvania, USA) tap water (to ensure conductivity) immediately before testing and transferred to polystyrene centrifuge tubes.

\section{Light obscuration}

Light obscuration (LO) was used to measure the number and size of oil droplets in the emulsion. In LO (Accusizer 780 Optical Particle Sizer, Nicomp PSS, Santa Barbara, California, USA), particles pass a detection zone whilst being illuminated by a laser beam. The method is also known as single particle optical sizing (SPOS). LO determines droplet sizes above $1 \mu \mathrm{m}$, and is therefore mostly interesting for capturing changes in the large diameter tail of the droplet size distribution.

In order to ensure that each droplet was detected separately, samples of remifentanil + propofol were diluted in MilliQ-water to a final volume of $40 \mathrm{~mL}$ in such a way that the number of droplets did not exceed the 9000 «particles»/ $\mathrm{mL}$ limit required by the instrument. The dilution factor varied from 1:200-1:28 000 (sample:water). Before analysis, the diluted sample contents were evenly distributed by gently shaking the centrifuge tube and resting for 60 seconds to 
allow air bubbles to escape. The same procedure was carried out for the controls of propofol.

Based on the measurements of number of the oil droplets of each size, PFAT5 was calculated as described by Gonyon et al. [14].

\section{Zeta potential}

Zeta potential of the propofol emulsion and mixed samples was measured using a Zetasizer nano series (Malvern instruments, Malvern, UK). The instrument was checked daily with a standard of known zeta potential $(-42 \pm 4.2 \mathrm{mV})$. Each sample was diluted in tap water to ensure sufficient conductivity in the solution.

\section{Multivariate data analysis}

All results are calculated and reported as mean \pm standard deviation (SD) using Microsoft Excel.

The correlation between variables was evaluated by principal component analysis (PCA) and partial least squares (PLS) regression using Unscrambler ${ }^{\circledR} 9.8$ (Camo ASA, Trondheim, Norway). PLS models were made based on (a) the full data matrix (144 samples), (b) based on all data immediately after mixing, i. e. excluding the measurements after 4 hours (72 samples), (c) based on all data for propofol $10 \mathrm{mg} / \mathrm{mL}$ (72 samples) and finally, (d) based on all data for propofol $10 \mathrm{mg} / \mathrm{mL}$ immediately after mixing, i. e. excluding the measurements after 4 hours (36 samples). Prior to analysis, the variation of each variable was scaled to unit variance (1/SD), and the models were cross-validated. The Unscrambler uses Jack-knifing to estimate the uncertainty of the regression coefficients of PLS [15], which for most practical reasons resembles $\alpha$ 0.05 . For more information on projection methods, see e. g. Esbensen et al. [16].

\section{Results}

The background noise in all tested centrifuge tubes and containers both filled with Milli-Q water were very low: $1.0 \pm 0.9$ particles $/ \mathrm{mL}$ measured immediately after filling and $2.5 \pm 2.4$ particles/mL measured after 4 hours. These values were well below the limit of background noise of 50 particles/mL, and demonstrate that the droplets analysed in this study had minimal risk of being influenced by particle contaminants originating from the water or the containers.

\section{$\mathrm{pH}$}

The $\mathrm{pH}$ of the reconstituted remifentanil solution was measured to be 3.5, which was in accordance with the information from the manufacturer [17]. The $\mathrm{pH}$ values of the three propofol formulations were around 7.5, as expected (Table 2). As shown in Table 3, the pH of the mixtures reflected their composition, i. e. when the mixing ratio contained high amounts of remifentanil the $\mathrm{pH}$ shifted towards acidic in contrast to samples with high amounts of propofol where the $\mathrm{pH}$ was neutral. Comparing $\mathrm{pH}$-values immediately after mixing and after 4 hours, a slight drop in $\mathrm{pH}$-values was seen for most samples and even some of the propofol controls.

\section{MDD, PI and zeta potential}

The MDD varied slightly between the different propofol formulations (Table 3), but was in the range between 165$200 \mathrm{~nm}$, well below the recommended cut-off of $500 \mathrm{~nm}$ for injectable emulsions. Small variations were observed between the various mixing ratios and the unmixed controls, e. g. statistically significant difference between $10 \mathrm{mg} /$ $\mathrm{mL}$ mixed with remifentanil versus the control for P-MCT1. However, we also found statistically significant differences between different batches of the same propofol formulation, i. e. control samples (data not shown).

All samples showed PI well below 0.1 indicating monodisperse droplet sizes. The zeta potential values of all samples ranged between -20 and $-40 \mathrm{mV}$ (data not shown). Of note is the fact that the zeta potential could be different within the range $(-30$ and $-40 \mathrm{mV})$ from one batch of the formulation to the next one.

\section{PFAT5}

All propofol control samples (unmixed propofol), showed PFAT5 values below the 0.05 limit both immediately and after 4 hours (Table 3). This indicated that all three propofol formulations were stable throughout the test period.

In all samples of propofol $10 \mathrm{mg} / \mathrm{mL}$ where remifentanil was added in abundance, PFAT5 values were above the recommended 0.05 limit 4 hours after mixing 
Table 3: Results of emulsion stability testing for propofol formulations (controls) and after mixing with remifentanil hydrochloride.

\begin{tabular}{|c|c|c|c|c|c|c|}
\hline \multirow{2}{*}{$\begin{array}{l}\text { Propofol formulation } \\
\text { and concentration }\end{array}$} & \multirow{2}{*}{$\begin{array}{r}\text { Mixing ratio, remifentanil } \\
\mathrm{HCl}+\text { propofol }\end{array}$} & \multirow{2}{*}{$\begin{array}{l}\text { Time after } \\
\text { mixing } h\end{array}$} & \multirow{2}{*}{$\begin{array}{r}\text { Light obscuration } \\
\text { PFAT5 }\end{array}$} & \multirow[t]{2}{*}{$\mathrm{pH}$} & \multicolumn{2}{|c|}{ Dynamic light scattering } \\
\hline & & & & & I.W. MDD nm & PI \\
\hline \multirow[t]{10}{*}{$P-M C T 110 \mathrm{mg} / \mathrm{mL}$} & Control & 0 & $0.004 \pm 0.002$ & 7.58 & $198.7 \pm 1,7$ & $0.062 \pm 0.026$ \\
\hline & & 4 & $0.004 \pm 0.002$ & 7.46 & & \\
\hline & $1+1$ & 0 & $0.014 \pm 0.008$ & 7.15 & $190.0 \pm 0.5$ & $0.050 \pm 0.017$ \\
\hline & & 4 & $0.015 \pm 0.009$ & 6.59 & & \\
\hline & $1+20$ & 0 & $0.004 \pm 0.002$ & 7.14 & $189.0 \pm 1.1$ & $0.058 \pm 0.025$ \\
\hline & & 4 & $0.004 \pm 0.002$ & 6.91 & & \\
\hline & $10+1$ & 0 & $0.032 \pm 0.003$ & 3.68 & $188.1 \pm 1.5$ & $0.046 \pm 0.020$ \\
\hline & & 4 & $0.780 \pm 0.507^{*}$ & 3.62 & & \\
\hline & $20+1$ & 0 & $0.026 \pm 0.003$ & 3.82 & $188.4 \pm 1.3$ & $0.056 \pm 0.015$ \\
\hline & & 4 & $0.216 \pm 0.096^{*}$ & 3.80 & & \\
\hline \multirow[t]{10}{*}{$P-M C T 120 \mathrm{mg} / \mathrm{mL}$} & Control & 0 & $0.008 \pm 0.002$ & 7.63 & $202.0 \pm 1.3$ & $0.045 \pm 0.027$ \\
\hline & & 4 & $0.007 \pm 0.003$ & 7.70 & & \\
\hline & $1+1$ & 0 & $0.046 \pm 0.016$ & 6.09 & $193.8 \pm 1.7$ & $0.068 \pm 0.022$ \\
\hline & & 4 & $0.072 \pm 0.022$ & 6.02 & & \\
\hline & $1+20$ & 0 & $0.007 \pm 0.004$ & 7.32 & $191.2 \pm 1.5$ & $0.061 \pm 0.022$ \\
\hline & & 4 & $0.006 \pm 0.005$ & 7.07 & & \\
\hline & $10+1$ & 0 & $0.129 \pm 0.034$ & 3.75 & $192.4 \pm 1.6$ & $0.082 \pm 0.010$ \\
\hline & & 4 & $0.590 \pm 0.200^{*}$ & 3.76 & & \\
\hline & $20+1$ & 0 & $0.062 \pm 0.017$ & 4.11 & $196.2 \pm 9.5$ & $0.059 \pm 0.027$ \\
\hline & & 4 & $0.651 \pm 0.278^{*}$ & 4.04 & & \\
\hline \multirow[t]{10}{*}{$P-M C T 210 \mathrm{mg} / \mathrm{mL}$} & Control & 0 & $0.013 \pm 0.002$ & 7.32 & $188.7 \pm 1.7$ & $0.079 \pm 0.022$ \\
\hline & & 4 & $0.015 \pm 0.005$ & 6.98 & & \\
\hline & $1+1$ & 0 & $0.072 \pm 0.017$ & 5.46 & $180.3 \pm 1.7$ & $0.083 \pm 0.019$ \\
\hline & & 4 & $0.255 \pm 0.076$ & 5.38 & & \\
\hline & $1+20$ & 0 & $0.017 \pm 0.005$ & 7.19 & $180.9 \pm 1.1$ & $0.083 \pm 0.013$ \\
\hline & & 4 & $0.014 \pm 0.001$ & 7.35 & & \\
\hline & $10+1$ & 0 & $0.048 \pm 0.001$ & 3.59 & $181.3 \pm 1.2$ & $0.085 \pm 0.010$ \\
\hline & & 4 & $0.248 \pm 0.113^{*}$ & 3.55 & & \\
\hline & $20+1$ & 0 & $0.026 \pm 0.007$ & 3.73 & $180.9 \pm 1.3$ & $0.090 \pm 0.020$ \\
\hline & & 4 & $0.560 \pm 0.178^{*}$ & 3.72 & & \\
\hline \multirow[t]{10}{*}{ P-MCT2 $20 \mathrm{mg} / \mathrm{mL}$} & Control & 0 & $0.012 \pm 0.008$ & 7.49 & $184.0 \pm 1.5$ & $0.080 \pm 0.006$ \\
\hline & & 4 & $0.011 \pm 0.007$ & 7.53 & & \\
\hline & $1+1$ & 0 & $0.122 \pm 0.048$ & 5.77 & $175.6 \pm 1.0$ & $0.095 \pm 0.011$ \\
\hline & & 4 & $0.555 \pm 0.129^{*}$ & 5.99 & & \\
\hline & $1+20$ & 0 & $0.014 \pm 0.004$ & 7.73 & $176.9 \pm 0.9$ & $0.089 \pm 0.021$ \\
\hline & & 4 & $0.013 \pm 0.004$ & 7.51 & & \\
\hline & $10+1$ & 0 & $0.167 \pm 0.078$ & 3.70 & $174.3 \pm 1.5$ & $0.078 \pm 0.013$ \\
\hline & & 4 & $0.945 \pm 0.089^{*}$ & 3.62 & & \\
\hline & $20+1$ & 0 & $0.024 \pm 0.003$ & 3.74 & $181.5 \pm 1.4$ & $0.085 \pm 0.012$ \\
\hline & & 4 & $0.269 \pm 0.096^{*}$ & 3.73 & & \\
\hline \multirow[t]{10}{*}{$P-L C T 10 \mathrm{mg} / \mathrm{mL}$} & Control & 0 & $0.013 \pm 0.003$ & 7.39 & $175.4 \pm 0.9$ & $0.078 \pm 0.024$ \\
\hline & & 4 & $0.013 \pm 0.003$ & 7.33 & & \\
\hline & $1+1$ & 0 & $0.031 \pm 0.013$ & 5.41 & $175.9 \pm 1.2$ & $0.075 \pm 0.013$ \\
\hline & & 4 & $0.026 \pm 0.000$ & 5.35 & & \\
\hline & $1+20$ & 0 & $0.015 \pm 0.002$ & 7.31 & $175.6 \pm 1.5$ & $0.059 \pm 0.018$ \\
\hline & & 4 & $0.015 \pm 0.002$ & 7.17 & & \\
\hline & $10+1$ & 0 & $0.033 \pm 0.014$ & 3.62 & $174.8 \pm 1.9$ & $0.069 \pm 0.021$ \\
\hline & & 4 & $0.095 \pm 0.035$ & 3.59 & & \\
\hline & $20+1$ & 0 & $0.017 \pm 0.001$ & 3.58 & $174.2 \pm 1.7$ & $0.080 \pm 0.020$ \\
\hline & & 4 & $0.037 \pm 0.013$ & 3.50 & & \\
\hline
\end{tabular}


Table 3: (continued)

\begin{tabular}{|c|c|c|c|c|c|c|}
\hline \multirow{2}{*}{$\begin{array}{l}\text { Propofol formulation } \\
\text { and concentration }\end{array}$} & \multirow{2}{*}{$\begin{array}{r}\text { Mixing ratio, remifentanil } \\
\qquad \mathrm{HCl}+\text { propofol }\end{array}$} & \multirow{2}{*}{$\begin{array}{l}\text { Time after } \\
\text { mixing } h\end{array}$} & \multirow{2}{*}{$\begin{array}{r}\text { Light obscuration } \\
\text { PFAT5 }\end{array}$} & \multirow[t]{2}{*}{$\mathrm{pH}$} & \multicolumn{2}{|c|}{ Dynamic light scattering } \\
\hline & & & & & I.W. MDD nm & PI \\
\hline \multirow[t]{10}{*}{$P-L C T 20 \mathrm{mg} / \mathrm{mL}$} & Control & 0 & $0.014 \pm 0.004$ & 7.09 & $168.2 \pm 1.9$ & $0.067 \pm 0.016$ \\
\hline & & 4 & $0.014 \pm 0.002$ & 7.22 & & \\
\hline & $1+1$ & 0 & $0.063 \pm 0.006$ & 5.17 & $169.6 \pm 0.9$ & $0.074 \pm 0.017$ \\
\hline & & 4 & $0.117 \pm 0.012$ & 5.15 & & \\
\hline & $1+20$ & 0 & $0.016 \pm 0.002$ & 7.08 & $168.5 \pm 1.3$ & $0.066 \pm 0.019$ \\
\hline & & 4 & $0.016 \pm 0.002$ & 7.04 & & \\
\hline & $10+1$ & 0 & $0.065 \pm 0.014$ & 3.73 & $166.5 \pm 1.0$ & $0.073 \pm 0.021$ \\
\hline & & 4 & $0.291 \pm 0.010^{*}$ & 3.69 & & \\
\hline & $20+1$ & 0 & $0.041 \pm 0.004$ & 3.57 & $167.5 \pm 1.0$ & $0.071 \pm 0.017$ \\
\hline & & 4 & $0.240 \pm 0.010^{*}$ & 3.62 & & \\
\hline
\end{tabular}

*Sample had to be diluted to meet the instrument's coincidence limit of 9000 particles $/ \mathrm{mL}$.

$\left(P-M C T 1=\right.$ Propolipid $^{\oplus}, P-M C T 2=$ Propofol-Lipuro ${ }^{\oplus}, P$-LCT $=$ Diprivan $^{\oplus}$, PFAT5 $=$ Percentage of fat residing in globules larger than $5 \mu \mathrm{m}$, I.W. $=$ Intensity weighted, $\mathrm{MDD}=$ Mean droplet diameter, $\mathrm{PI}=$ Polydispersity index. Mean \pm standard deviation).

(long contact time); this happened immediately after mixing in samples of propofol $20 \mathrm{mg} / \mathrm{mL}$ irrespective of the mixing ratios of remifentanil and propofol (Table 3).

Principal component analysis (PCA) showed that PFAT5 displayed a positive correlation with remifentanil content in the mixing ratio (high value of ratio and high PFAT5), time after mixing (high value of time and high PFAT5) and negative correlation with $\mathrm{pH}$-values of the sample (high value of $\mathrm{pH}$ located on the opposite side of origo from PFAT5) (Figure 1(A)). Propofol concentration was somewhat correlated with PFAT5, but to a lesser extent than the other factors as shown by the loading located much closer to origo on the first two principal components. The distribution of samples in the score plot showed three distinct groups along the first principal component (PC1). The group with the highest positive values on PC1 (right end of the x-axis) displayed a positive correlation with PFAT5, whereas the group with the highest negative values (left end of the $\mathrm{x}$-axis) displayed a negative correlation with PFAT5. This group displayed a positive correlation with the $\mathrm{pH}$ values of the samples. It should be noted that all propofol formulations are present in all three groups; however, an overrepresentation of $P$-MCT2 (green color in Figure 1(B)) might be seen in the group with high PFAT5-values.

The influence of each variable on PFAT5 was analysed by PLS regression (Figure 2). The regression coefficients from PLS models of all factors containing all samples (full matrix) showed that propofol concentration, mixing ratio, mixing time and $\mathrm{pH}$ had a significant influence on PFAT5 (Figure 2(A)). High propofol concentration $(20 \mathrm{mg} / \mathrm{mL})$, long contact time ( $4 \mathrm{~h}$ after mixing) and low
pH-values (high amounts of remifentanil) were positively correlated with high PFAT5 values.

Another interesting finding was that $P$-LCT showed a negative correlation with PFAT5 values whereas P-MCT2 showed a positive one. No significant correlation between $P$-MCT1 and PFAT5 values was identified.

Since long contact time between remifentanil and propofol seemed to have a detrimental effect on the PFAT5, it was interesting to investigate the data from the immediate time point separately. Therefore, data obtained $4 \mathrm{~h}$ after mixing were left out and a new PLS model was made for the PFAT5 measured immediately after mixing. The regression coefficients showed the same correlations with PFAT5 as before (Figure 2(B)), even though most of the PFAT5 values for immediate samples were within the 0.05 limit (Table 3). Due to the identified correlations of high propofol concentration and long contact time with high PFAT5, PLS analyses were performed on the data set of propofol $10 \mathrm{mg} / \mathrm{mL}$ separately (Figure 2(C)), and propofol $10 \mathrm{mg} / \mathrm{mL}$ immediately after mixing separately (Figure 2(D)). Similar trends of the regression coefficients were again seen to correlate to high PFAT5.

\section{Discussion}

Propofol is very slightly soluble in water, and is, therefore formulated as an oil-in-water emulsion. Injectable emulsions are composed of oil droplets in water stabilized by one or more emulsifiers. The droplets have a charged surface securing the necessary repulsion between the drop- 
A)

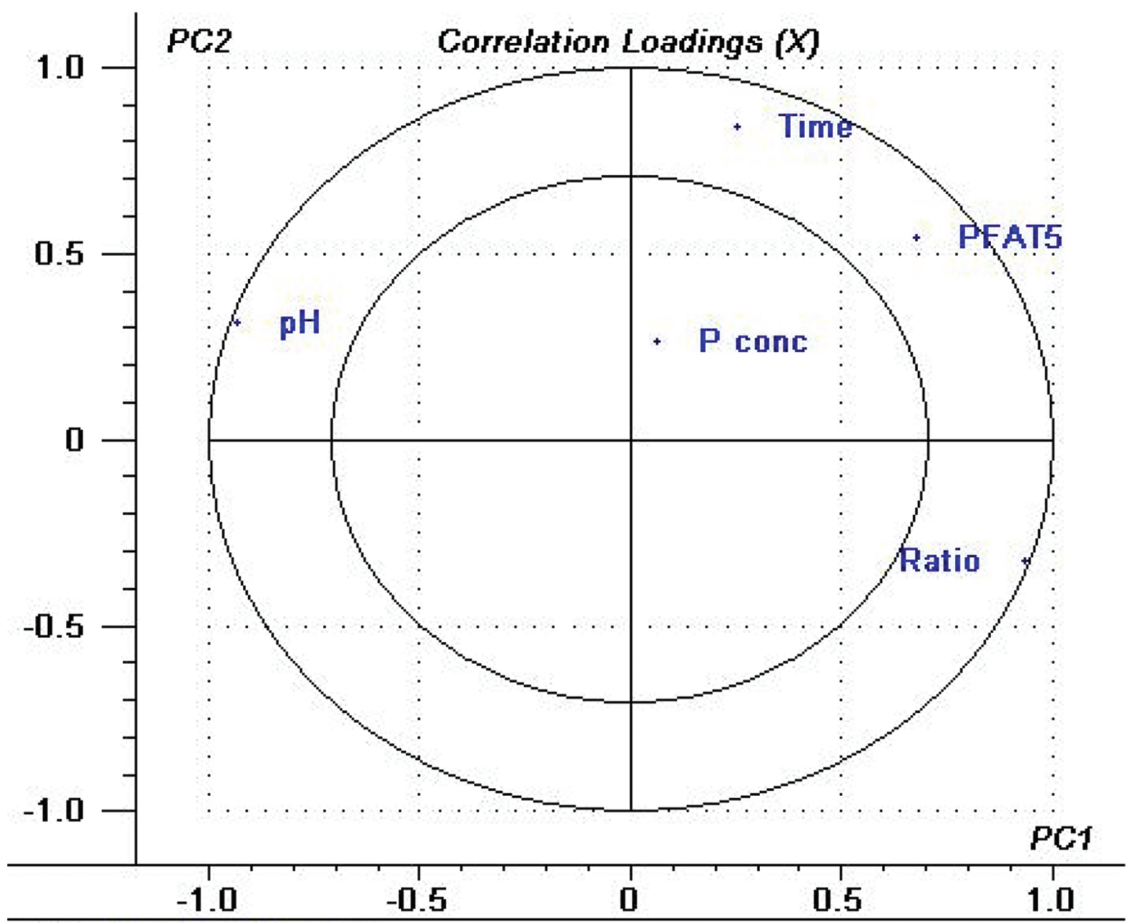

PCA, X-expl: $45 \%, 25 \%$

B)

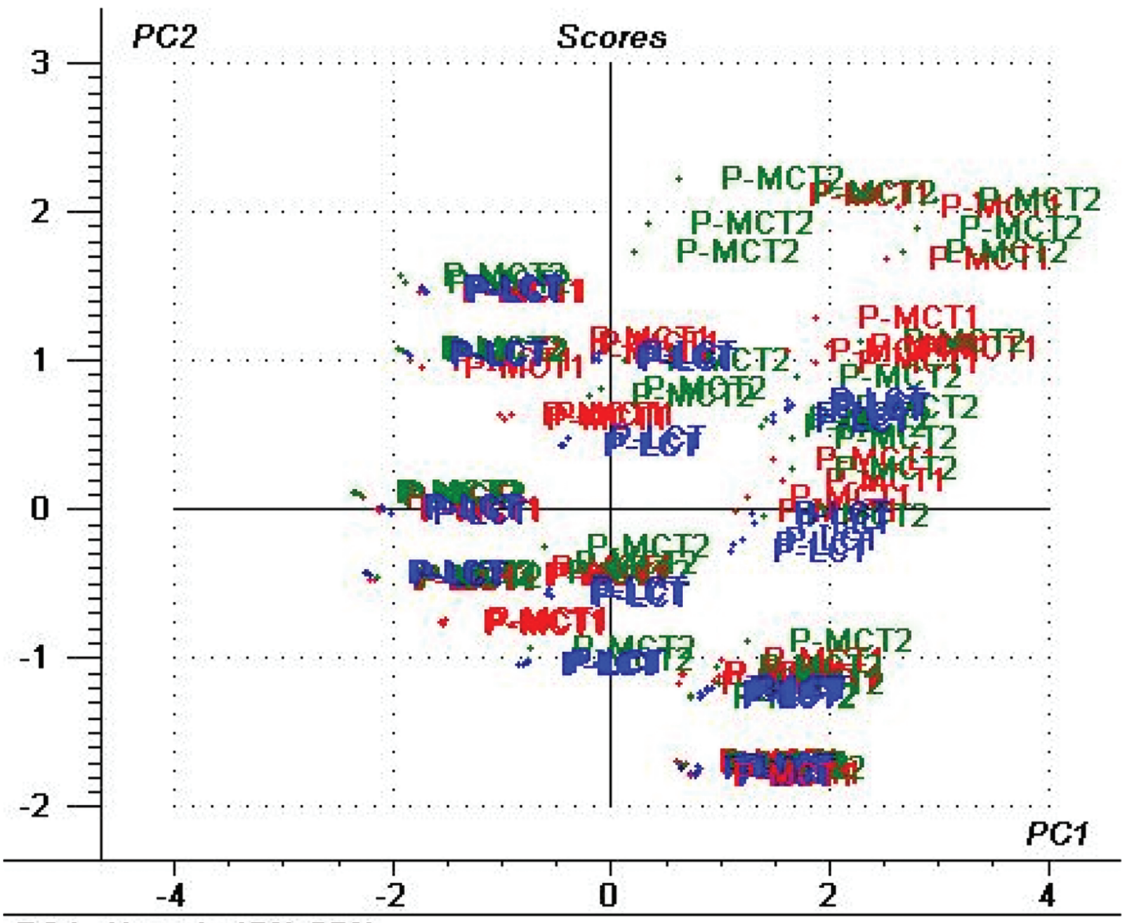

PCA, X-expl: $45 \%, 25 \%$

Figure 1: Results from a principal component analysis (PCA) of PFAT5 (percentage of fat residing in globules larger than $5 \mu \mathrm{m}$ ) for all mixtures of remifentanil with the various propofol formulations. (A) correlation loading plot showing the correlation between the factors. (B) score plot showing the projections of the samples.

$P-M C T 1=$ Propolipid, $P$-MCT2 $=$ Propofol-Lipuro, $P$-LCT $=$ Diprivan, $\mathrm{P}$ conc $=$ propofol concentration, Ratio $=$ mixing ratio of remifentanil and propofol, Time $=$ time after mixing propofol with remifentanil, determined PFAT5 and $\mathrm{pH}$. 


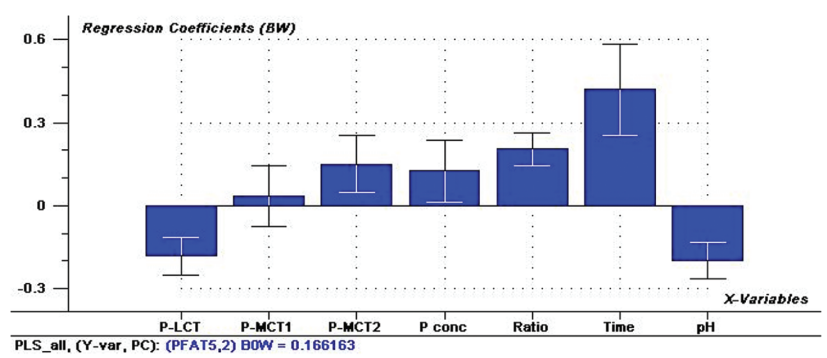

C)

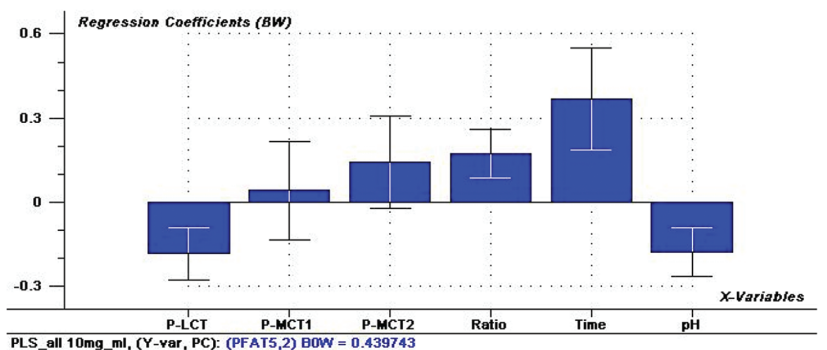

B)

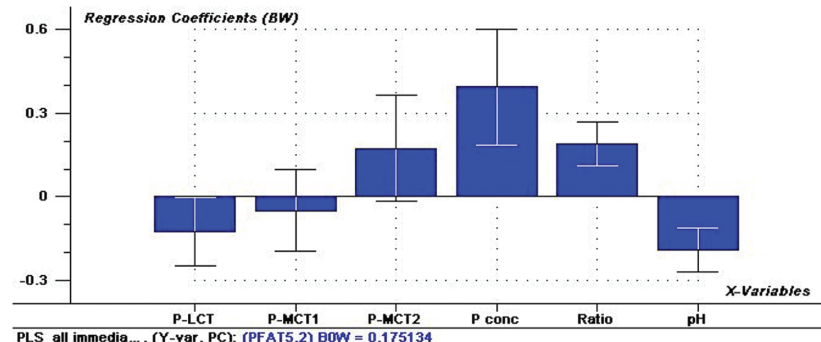

D)

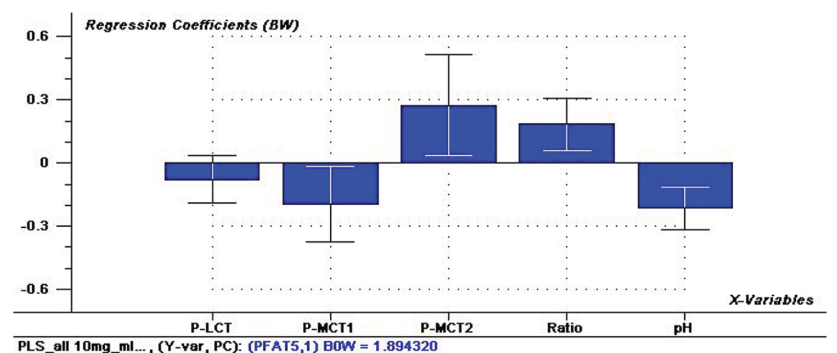

Figure 2: Balanced and weighted (BW) regression coefficients from partial least squares (PLS1) regression analyses of investigated variables on the measured PFAT5 (percentage of fat residing in globules larger than $5 \mu \mathrm{m}$ ) in mixtures of propofol and remifentanil. Significant factors are those with error bars not crossing zero. Propofol formulation ( $P$-MCT1 = Propolipid, $P$-MCT2 = Propofol-Lipuro, $P$-LCT = Diprivan), $P$ conc $=$ propofol concentration, Ratio $=$ mixing ratio of remifentanil and propofol, Time $=$ time after mixing propofol with remifentanil, determined $\mathrm{pH}$. (A) Full data matrix. (B) All formulations but only data immediate after mixing, i. e. excluding measurements of PFAT5 4 hours after mixing. (C) All formulations of $10 \mathrm{mg} / \mathrm{mL}$ propofol. (D) All formulations of $10 \mathrm{mg} / \mathrm{mL}$ propofol immediate after mixing i. e. excluding measurements of PFAT5 4 hours after mixing.

lets, thereby, avoiding coalescence and phase separation [18]. Emulsions are sensitive to physicochemical changes, such as changes in $\mathrm{pH}$, temperature or variation in electrolyte contents (e.g. oppositely charged counter ions). In a destabilised emulsion, an increase in droplet size will occur $[14,19,20]$. Since intravenous infusion of enlarged droplets encompass potentially serious risks, droplet stability is essential for injectable emulsions [2-9].

Knowledge on propofol emulsion stability when coadministered with remifentanil is sparse. None of the few studies that have been conducted is able to show conclusive evidence about the compatibility between propofol and remifentanil or lack of such [10-12]. No studies have analysed both $10 \mathrm{mg} / \mathrm{mL}$ and $20 \mathrm{mg} / \mathrm{mL}$ propofol even though both concentrations are used regularly in the clinical setting. Neither have a direct comparison of different propofol formulations been carried out.

To capture possible changes in emulsion stability we used different analysis methods, including dynamic light scattering, measurements of $\mathrm{pH}$ and zeta potential, and analyses of size and number of large oil droplets using light obscuration followed by calculation of PFAT5. Even though significant differences in MDD could sometimes be identified between the control and mixed samples, despite all being well below $500 \mathrm{~nm}$, this should not be directly interpreted as incompatibility. The mean droplet size is not the best parameter to assess changes in stability due to droplet growth [21]. As mentioned above, the first signs of droplet growth appears in the large diameter tail of the size distribution, and a considerable amount of large droplets will have to be formed before it can be recognized as an increased MDD. In the current study, MDD and PI were measured to keep an eye on the bulk properties, which was useful since small differences could be observed between different batches of the same formulation, but PFAT5 is the parameter that provides the information on the large diameter tail region. Defined as the percentage of fat droplets larger than $5 \mu \mathrm{m}$, increased PFAT5 values suggest destabilisation of emulsion. According to USP <729> PFAT5 values should lie below 0.05 (i. e. $5 \%$ of droplets below $5 \mu \mathrm{m}$ in size) to ensure emulsion stability, from an infusion safety perspective [1].

A slight decrease in $\mathrm{pH}$-values was observed for most samples after 4 hours contact time. It is known from literature that lipid emulsions, when exposed to air, may undergo oxidative processes releasing free fatty acids and lowering $\mathrm{pH}$ [22]. Steger and Mühlebach showed that emulsions based on LCT and LCT/MCT 
degrade at different rates under light exposure at room temperature [22]. Therefore, lipid phase stability should not be forgotten when evaluating emulsion stability.

The experimental area was spanned out with the aim of making the results as clinically relevant as possible. The maintenance dose of propofol is $5-150 \mu \mathrm{g} / \mathrm{kg} / \mathrm{min}$ and that of remifentanil is $0.008-0.25 \mu \mathrm{g} / \mathrm{kg} / \mathrm{min}$. This is in concordance with local guidelines at Oslo University Hospital, Norway [23], the American College of Critical Care Medicine's revised guideline "Clinical practice guidelines for the management of Pain, Agitation and Delirium (PAD)" [24], and information provided in the section General anesthesia: maintenance, in UpToDate [25]. This gives a wide range of possibilities of mixing ratios when adjusting the dose according to the response of the patient. To also accommodate for the possible scenario of bolus doses, the mixing ratios spanned from $20+1$ to $1+20$ for remifentanil: propofol. This was discussed with local clinicians, taking anesthesiology both during surgery and at intensive care into account.

The infusion rates of the drugs are determined by the desired level of sedation and the contact time of the drugs in the i.v. line is therefore difficult to predict. We therefore, analysed all samples immediately after mixing and 4 hours after mixing remifentanil and propofol. We did not identify any values of PFAT5 above the recommended limit of 0.05 immediately after mixing with remifentanil for all three formulations at a propofol concentration of $10 \mathrm{mg} / \mathrm{mL}$, but elevated PFAT5 values were seen in most samples after 4 hours (Table 3). This is indicative of emulsion destabilisation at long contact times, which can lead to adverse implications for the patient during surgery that last longer or in cases where the infusion rates are very slow, e. g. for children. In fact, the infusion rates in paediatric patients might be much longer with contact times of the drugs exceeding the experimental conditions covered by this study.

For all three formulations, the high propofol concentration $(20 \mathrm{mg} / \mathrm{mL})$ was more prone to the formation of larger droplets after mixing with remifentanil (Table 3). This phenomenon was even more marked after 4 hours, but also significant immediately after mixing (Figure 2). The direct correlation of PFAT5 with propofol concentration, and samples where remifentanil was in abundance and $\mathrm{pH}$ was low (i. e. high ratio of remifentanil) indicated that these factors influence the stability of propofol emulsions. Gersonde et al. also reported high PFAT5 values for two MCT-based propofol formulations (Propofol-Lipuro and Propofol MCT) at $20 \mathrm{mg} / \mathrm{mL}$ upon mixing with remifentanil [11]. They measured the samples 24 hour after mixing. Nemec et al. found an aggregate formation immediately after mixing equal parts remifentanil either with Propofol LCT $2 \%(20 \mathrm{mg} / \mathrm{mL})$ or Propofol MCT 1\% (10 mg/mL) and recommended not to mix remifentanil directly with propofol [10].

Our regression models suggested that the different propofol formulations responded differently to mixing with remifentanil. It seemed like the P-MCT2 formulation (Propofol-Lipuro) was positively correlated with PFAT5 and associated with high values of PFAT5 immediately after mixing with remifentanil and also after 4 hours whereas this was not the case for the other MCT-formulation (Propolipid) or the LCT-formulation (Diprivan) (Figure 2(AD)). This deviates from the reports from the manufacturer $B$. Braun, as they did not see any sign of emulsion destabilisation in their analysis of their MCT-formulation (PropofolLipuro 1\%) mixed with remifentanil performed after one hour storage. Their mixing ratios were $10+1,1+1$ and $1+10$ [26]. For P-LCT we identified high PFAT5 values for both concentrations but only 4 hours after mixing with remifentanil (Table 3). Humbert-Delaloye et al. reported no sign of emulsion destabilisation when visually inspecting Disoprivan (LCT) $20 \mathrm{mg} / \mathrm{mL}$ mixed with remifentanil in glucose $(50 \mathrm{mg} / \mathrm{mL})$ in the concentration $0.12 \mathrm{mg} / \mathrm{mL}$ [12], but visual examination is not an accurate method to identify early signs of emulsion destablilisation . Even though our findings suggest that different propofol formulations can react differently upon mixing with remifentanil, the difference in the lipid phase alone (MCT vs LCT) could not explain these differences; the complete formulation and/or the manufacturing process are the most likely determining factors.

We did not see any high values of PFAT5 in the mixing ratio of $1+20$, i.e. propofol in abundance, neither immediately nor 4 hours after mixing. In intensive care units, propofol $20 \mathrm{mg} / \mathrm{mL}$ is frequently used, and at this concentration, we found high PFAT5 values for all three propofol formulations, both immediately and after 4 hours, in mixing ratios $1+1$ and $10+1$, that is when remifentanil was present in equal parts or in abundance. This demonstrates that high propofol concentrations are more sensitive to reactions with remifentanil and caution is advised when remifentanil is present in equal parts or in abundance.

Stewart et al. concluded that from a chemical stability perspective of the drugs, it was safe to mix remifentanil and propofol in a polypropylene syringe and store for up to 36 hours, but mixed in the same polyvinylchloride bag the mixture should be used within 3 hours [27]. Our results showed that from a physical compatibility perspective it is not safe to store remifentanil mixed with propofol in the same container, such as premixing in a syringe, since both long contact time and mixing ratios of remifentanil $>$ propofol increased the risk of emulsion 
instability. In fact, the importance of always including an emulsion stability test in compatibility studies involving propofol was emphasized by Cherin and Smiler in a letter to the editor as a response to the study of Stewart et al. [28]. If premixing remifentanil and propofol is desired, propofol $10 \mathrm{mg} / \mathrm{mL}$ should be used, the ratio of remifentanil to propofol should be dominated by propofol, and the mixture should be utilised within 4 hours, as we lack stability data beyond 4 hours contact time.

Our findings need to be interpreted with the following limitations and strengths in mind: The samples had to be strongly diluted in order to be analysed. This may have affected the physicochemical properties of the emulsions since flocculates/aggregates that otherwise would gradually coalesce and destabilise the emulsion may have been broken up by the dilution process. However, the control samples of propofol alone were diluted to the same extent. Another limitation is that by performing sample preparation and analysis in the lab environment and not in a classified clean room, the risk of particle contamination from the surroundings as well as from the equipment could not be entirely eliminated, although particle content in the MilliQ-water and main containers were checked. Particles, if in the right size range, could be interpreted as emulsion droplets. The clinical relevance of a rigid cut off limit of $5 \mu \mathrm{m}$ could be discussed [29]. Oil droplets are more flexible and malleable than solid rigid particles, such as precipitates of crystals, and might be better tolerated by the human body. Furthermore, the $5 \%$ threshold of the acceptable amount of enlarged droplets can also be discussed, and it might be worth mentioning that the PFAT5 monography of the USP does not have an equivalent in the European Pharmacopeia. Emulsion stability was only analysed at two time points, immediately after mixing and 4 hours after mixing, and there will be clinical scenarios that were not accounted for in this study. Infusion rates, especially for the paediatric population, could exceed 4 hours, and as shown, extended contact times could lead to emulsion destabilisation. Last, but not least, our study was set up to simulate a Y-site administration of remifentanil and propofol and could not be directly converted to the dynamic situation when drugs are co-administered via the same i.v. line.

\section{Conclusions}

Differences in emulsion stability depending on the propofol formulation were identified under the most extreme test conditions in our study (long contact times and excess of remifentanil in the mixing ratio). The differences could not be explained by type of propofol lipid phase alone. Nevertheless, all tested propofol formulations were found to be stable after mixing with remifentanil in ratios where propofol was in abundance. Our results indicate that coadministration of remifentanil and propofol $20 \mathrm{mg} / \mathrm{mL}$ in the same i.v. line when remifentanil is present in equal parts or in abundance should preferably be avoided. Based on the results after 4 hours contact time, i. e. development of larger droplets and a drop in $\mathrm{pH}$, premixing of propofol and remifentanil in syringes or as admixtures should, if possible, be avoided. Since destabilisation of the emulsion may increase the risk of large fat droplet infusion, we recommend taking differences in emulsion stability between propofol formulations into account when switching between different propofol products.

\section{Abbreviations}

$\begin{array}{ll}\text { PFAT5 } & \text { Percentage of fat droplets larger than } 5 \mu \mathrm{m} \\ \text { I.W. } & \text { Intensity weighted } \\ \text { MDD } & \text { Mean droplet diameter } \\ \text { PI } & \text { Polydispersity index } \\ \text { USP } & \text { United States Pharmacopeia } \\ \text { DLS } & \text { Dynamic light scattering } \\ \text { LO } & \text { Light obscuration } \\ \text { PCA } & \text { Principal component analyses } \\ \text { PLS } & \text { Partial least squares } \\ \text { MCT } & \text { Medium chain triglycerides } \\ \text { LCT } & \text { Long chain triglycerides } \\ \text { HCl } & \text { Hydrochloride } \\ \text { i.v. } & \text { intravenous } \\ \text { SmPC } & \text { Summary of product characteristics } \\ \text { P-MCT1 } & \text { Propolipid }{ }^{\circledR} \\ P-M C T 2 & \text { Propofol-Lipuro }{ }^{\circledR} \\ \text { P-LCT } & \text { Diprivan }{ }^{\circledR}\end{array}$

Acknowledgements: We would like to extend our thanks to The South-Eastern Norway Regional Health Authority for the funding (project number 2018096), all nurses and physicians at the division of emergency, critical care and surgery at Oslo University Hospital. Thanks to Vigdis Staven Berge (Ph.D), Jørgen Brustugun (Ph.D), both at Oslo Hospital Pharmacy, and Kaveh Teimori (MPharm at Sahlgrenska University Hospital, Gothenburg, Sweden) for all invaluable advices. Thanks to Tove Larsen (engineer at University of Oslo) for all your help in the laboratory.

Research funding: This work was supported by SouthEastern Norway Regional Health Authority, project number 2018096. The funder had no role in the study design, data 
collection or analysis, decision to publish, or the preparation of the manuscript.

Conflict of interest statement: The authors state no conflict of interest. The authors have read the journal's Publication ethics and publication malpractice statement available at the journal's website and hereby confirm that they comply with all its parts applicable to the present scientific work.

\section{References}

1. United States Pharmacopeia. Generell chapter: <729> Globule size distribution in lipid injectable emulsions. In: USP44-NF37. Available at: https://www.uspnf.com. Accessed: 15 Apr 2019.

2. Hippalgaonkar K, Majumdar S, Kansara V. Injectable lipid emulsions-advancements, opportunities and challenges. AAPS PharmSciTech 2010;11:1526-40.

3. Reedy JS, Kuhlman JE, Voytovich M. Microvascular pulmonary emboli secondary to precipitated crystals in a patient receiving total parenteral nutrition: a case report and description of the high-resolution CT findings. Chest 1999;115:892-5.

4. McNearney T, Bajaj C, Boyars M, Cottingham J, Haque A. Total parenteral nutrition associated crystalline precipitates resulting in pulmonary artery occlusions and alveolar granulomas. Dig Dis Sci 2003;48:1352-4.

5. Akhtar S. Fat embolism. Anesthesiol Clin 2009;27:533-50.

6. Klang MG. PFAT5 and the evolution of lipid admixture stability. J Parenteral Enteral Nutr 2015;39:67S-71S.

7. Wretlind A. Invited review: development of fat emulsions. J Parenteral Enteral Nutr 1981;5:230-5.

8. Jack T, Boehne M, Brent BE, Hoy L, Köditz H, Wessel A, et al. In-line filtration reduces severe complications and length of stay on pediatric intensive care unit: a prospective, randomized, controlled trial. Intensive Care Med 2012;38:1008-16.

9. Benlabed M, Perez M, Gaudy R, Genay S, Lannoy D, Barthelemy C, et al. Clinical implications of intravenous drug incompatibilities in critically ill patients. Anaesth Crit Care Pain Med 2019;38:173-80.

10. Nemec K, Germ E, Schulz-Siegmund M, Ortner A, Ortner A. The effect of nimodipine, fentanyl and remifentanil intravenous products on the stability of propofol emulsions. Die Pharmazie 2009;64:94-7.

11. Gersonde F, Eisend S, Haake N, Kunze T. Physicochemical compatibility and emulsion stability of propofol with commonly used analgesics and sedatives in an intensive care unit. Eur J Hosp Pharm 2017;24:293-303.

12. Humbert-Delaloye V, Berger M, Voirol P, Pannatier A. In vitro compatibility of remifentanil hydrochloride and sufentanil citrate with selected drugs. Eur J Hosp Pharm 2012;19:57-64.

13. Müller RH, Benita S, Böhm BH. Emulsions and nanosuspensions for the formulation of poorly soluble drugs. Nanosuspensions: Stuttgart Medpharm, 1998:149-74.
14. Gonyon T, Patel P, Owen H, Dunham AJ, Carter PW. Physicochemical stability of lipid injectable emulsions: correlating changes in large globule distributions with phase separation behavior. Int J Pharm 2007;343:208-19.

15. Martens H, Martens M. Modified Jack-knife estimation of parameter uncertainty in bilinear modelling by partial least squares regression (PLSR). Food Qual Prefer 2000;11:5-16

16. Esbensen KH, Guyot D, Westad F, Houmoller LP. Multivariate data analysis: in practice: an introduction to multivariate data analysis and experimental design. Oslo: Camo Process AS, 2002.

17. Ultiva. Summary of Product Characteristics (SmPC), 2019. Available at: https://www.medicines.org.uk/emc/product/ 795/smpc. Accessed: 26 Feb 2019.

18. Washington C. Stability of lipid emulsions for drug delivery. Adv Drug Deliv Rev 1996;20:131-45.

19. Baker MT, Naguib M. Propofol: the challenges of formulation. Anesthesiology 2005;103:860-76.

20. Driscoll DF. Lipid injectable emulsions: pharmacopeial and safety issues. Pharm Res 2006;23:1959.

21. Staven V, Wang S, Gronlie I, Tho I. Development and evaluation of a test program for Y-site compatibility testing of total parenteral nutrition and intravenous drugs. Nutr J 2016;15:29.

22. Steger PJ, Muhlebach SF. In vitro oxidation of i.v. lipid emulsions in different all-in-one admixture bags assessed by an iodometric assay and gas-liquid chromatography. Nutrition (Burbank, Los Angeles County, Calif). 1997;13:133-40.

23. Oslo University Hospital. Intensivpasienter - smerte, uro/agitasjon og delirium, voksne. Available at: https://ehandboken. ous-hf.no/document/56319/fields/23. Updated: 25 Nov 2018; Accessed: 4 Jul 2019.

24. Barr J, Fraser GL, Puntillo K, Ely EW, Gelinas C, Dasta JF, et al. Clinical practice guidelines for the management of pain, agitation, and delirium in adult patients in the intensive care unit. Crit Care Med 2013;41:263-306.

25. King A. General anesthesia, maintenance. In: Nussmeie NA, Girish JP, editors. UpToDate; Available at: https://www. uptodate.com/contents/general-anesthesia-maintenance? search $=$ General\%20anesthesia;\%20Maintanance \&source= search_result\&selectedTitle $=1 \sim 150 \&$ usage_type $=$ default $\&$ dis play_rank=1. Updated 29 Aug 2018; Accessed: 4 Jul 2019.

26. Klingmüller M. Evaluation of the compatibility of Propofol-Lipuro $1 \%$ with remifentanil and ringerfundin. Ecotox, 2012. Report No.: E1207-6-082813. Sponsered by BBraun Medical AG.

27. Stewart JT, Warren FW, Maddox FC, Viswanathan K, Fox JL. The stability of remifentanil hydrochloride and propofol mixtures in polypropylene syringes and polyvinylchloride bags at 22 degrees-24 degrees C. Anesth Analg 2000;90:1450-1.

28. Chernin EL, Smiler B. Any propofol compatibility study must include an emulsion stability analysis. Anesth Analg 2000;91:1307-8.

29. Driscoll DF, Nehne J, Peterss H, Franke R, Bistrian BR, Niemann W. The influence of medium-chain triglycerides on the stability of allin-one formulations. Int J Pharm 2002;240:1-10. 